\title{
Evolving Techniques in RSI: Can the Choice of Induction Agent Matter in Securing a Definitive Airway in Emergency Settings?
}

\author{
Bindu George $^{1} \odot$, Nayanthara Joachim ${ }^{2}$ (] \\ Keywords: Airway management, Endotracheal intubation, Etomidate, Intravenous anesthetics, Ketamine, Rapid sequence intubation. \\ Indian Journal of Critical Care Medicine (2022): 10.5005/jp-journals-10071-24100
}

The most important task of an emergency physician managing an acutely unstable patient is to secure the airway. ${ }^{1}$ Rapid sequence induction (RSI) is the technique of choice for emergency airway management of all patients except in anticipated difficult airways. Prevention of aspiration, rapid and safe achievement of intubation, and preparation for the possibility of failure of securing the airway are the immediate goals.

In the prehospital setting, there is variable access to expertise and equipment. There is always increased mortality associated with prehospital intubation caused by suboptimal intubation performance.

The classical approach to RSI, as advocated by Stept and Safar describe certain essential features: ${ }^{2}$

- Preoxygenation with $100 \%$ oxygen.

- Administration of a predetermined induction dose.

- Application of cricoid pressure and avoid bag and mask ventilation.

- Intubation with a cuffed tracheal tube.

- Specialized equipment for management in the event of a failed intubation.

A wide-bore intravenous (IV) connected to a running intravenous fluid ensures quick circulation of drugs to the brain. Over the years, the practice of RSI has evolved with new drugs and equipment. Modified or evolving RSI is a term used to describe variation from classic RSI like using different intravenous induction agents, a combination of agents with or without opioids, titration of doses of induction agents, neuromuscular blockers other than succinylcholine, and gentle mask ventilation during apnea. Modified approaches tend to trade on an increased risk of aspiration for other benefits like preventing hypoxia, respiratory acidosis, hemodynamic instability, awareness, slow induction, etc.

Historically, classical RSI has employed thiopentone as an induction agent and succinylcholine as the neuromuscular blocking agent. It took many years to introduce newer induction agents like propofol, etomidate, ketamine, and midazolam. ${ }^{3}$ Induction agents are integral to perform RSI. Instead of titrating to effect, RSI involves weight-based fixed doses of induction agents.

Ideal intravenous induction agents used for RSI should facilitate the loss of consciousness in one arm brain circulation time, minimize the time from loss of consciousness to intubation, create optimum hemodynamics, and avoid secondary insults to injured organs. Depending on the clinical circumstances, the intensivist may utilize a combination of induction and preinduction agents.
1,2Department of Anaesthesiology, St John's Medical College and Hospital, Bengaluru, Karnataka, India

Corresponding Author:Bindu George,Department of Anaesthesiology, St John's Medical College and Hospital, Bengaluru, Karnataka, India, Phone: +91 9342552324, e-mail: bindu575@gmail.com

How to cite this article: George B, Joachim N. Evolving Techniques in RSI: Can the Choice of Induction Agent Matter in Securing a Definitive Airway in Emergency Settings? Indian J Crit Care Med 2022;26(1):15-17.

Source of support: Nil

Conflict of interest: None

Specific induction agents offer advantages in certain emergency clinical scenarios. Etomidate is used in the presence of hemodynamic instability and poor cardiac function. Ketamine with its unique sympathomimetic and bronchodilating capabilities is indicated in status asthmaticus and to an extent in patients with hemodynamic instability. Propofol is preferred in isolated traumatic brain injuries with status epilepticus. Combination of propofol and ketamine, ketofol, is also used in hemodynamically compromised patients. ${ }^{4}$

Ketamine, a dissociative anesthetic agent acts on the N-methyl-Daspartate receptor complex causing neuro inhibition and anesthesia. ${ }^{5}$ Hemodynamic effects of ketamine are predominantly mediated through catecholamine release. So catecholamine-depleted critically ill patients may be unprotected from unbalanced direct myocardial depressant effects of ketamine. ${ }^{6}$ Ketamine also acts on receptors like opioid and monoaminergic receptors. Ketamine causes an increase in blood pressure, heart rate, and cardiac output in a biphasic manner. It produces a direct cardiodepressive or negative inotropic effect. ${ }^{7}$ Its stimulatory effect on the sympathetic system, in turn, causes the systemic release of catecholamines, inhibition of vagal nerves, and inhibition of norepinephrine (NE) uptake at peripheral nerves. Direct sympathetic stimulation makes ketamine an attractive option for induction in unstable patients with hypovolemia, sepsis, or shock. But the intrinsic myocardial depressant effect of ketamine may predominate in patients with massive stress-induced catecholamine depletion. Hence, the use of ketamine does not obviate the need for appropriate resuscitation in shock. ${ }^{9}$

Etomidate is an imidazole-derived sedative-hypnotic that acts on the gamma aminobutyric acid receptor complex. Its unique property is its hemodynamic stability. However, its use was tempered by reports that the drug can cause inhibition of steroid synthesis after a single dose. ${ }^{8}$ Its use has again expanded after the

(0) The Author(s). 2022 Open Access This article is distributed under the terms of the Creative Commons Attribution 4.0 International License (https://creativecommons. org/licenses/by-nc/4.0/), which permits unrestricted use, distribution, and non-commercial reproduction in any medium, provided you give appropriate credit to the original author(s) and the source, provide a link to the Creative Commons license, and indicate if changes were made. The Creative Commons Public Domain Dedication waiver (http://creativecommons.org/publicdomain/zero/1.0/) applies to the data made available in this article, unless otherwise stated. 
rediscovery of its beneficial physiologic profile and lack of novel reports on significant adrenal suppression. Hemodynamic stability with etomidate is due to its lack of effect on the sympathetic nervous system and on the function of baroreceptors. Minimal effects on cardiovascular function set it apart from other rapid onset IV anesthetics. The myocardial oxygen supply-demand ratio is well maintained, though it causes a minimal decrease in peripheral vascular resistance. One disadvantage is that etomidate lacks analgesic efficacy and hence needs to be combined with opiates to prevent hemodynamic stress response during laryngoscopy and intubation. This is vital in patients with hypertension, cardiac diseases, and raised intracranial pressure (ICP). Its dose-dependent reversible inhibition of enzyme 11 b hydroxylase, result in decreased biosynthesis of cortisol. Etomidate can cause adrenocortical suppression at a concentration less than $10 \mathrm{ng} / \mathrm{mL}$, much lower than its therapeutic level for inducing hypnosis. ${ }^{8}$ Etomidate-associated suppression of adrenal steroidogenesis lasts up to 4-12 hours. There is no conclusive evidence that etomidate increases mortality after a single dose. ${ }^{9,10}$ Other limitations of etomidate are myoclonus, pain on injection, and postoperative nausea and vomiting (PONV). Its new derivative methoxycarbonyl etomidate is without adrenocortical suppression. ${ }^{8}$

On this issue, Saurabh et al. have done a systematic review and meta-analysis on etomidate vs ketamine for induction during RSI. The aim of this review and meta-analysis was to compare the safety of etomidate and ketamine as induction agents for RSI and to see if either agent were superior in critically ill patients in the emergency department and prehospital settings. This was with respect to postinduction hypotension and first-pass intubation success during RSI.

Of 87 records identified, 9 were eligible, all of which had a low to moderate risk of overall bias. Six studies, including 12,060 patients from prehospital emergency medical services, air medical transport, and emergency department settings compared postinduction hypotension incidence, between etomidate and ketamine groups. The meta-analysis showed that etomidate use was associated with decreased risk of postinduction hypotension compared to ketamine (OR: $0.53 ; 95 \% \mathrm{Cl}: 0.31-0.91 ; \mathrm{l}^{2}=68 \%$ ). Seven studies, including 15,574 patients, reported on the rate of first-pass intubation success with etomidate vs ketamine. In the pooled analysis, no differences were seen in first-pass intubation success during RSI using etomidate vs ketamine.

A comprehensive review is not available in the literature regarding the impact of the choice of induction agent for RSI on the success of the procedure and hemodynamic stability with the use of different induction agents. It is commendable that Saurabh et al. are one of the first researchers to do a systematic review on evolving RSI based on the choice of induction agents, which can affect morbidity in emergency settings.

Out of the six studies for postinduction hypotension, two were RCTs and four were retrospective observational studies. Authors agree that the certainty of evidence from this meta-analysis is considered moderate because of concerns for bias due to the largely observational and retrospective nature of the study, which is true and a conclusion is limited.

The use of logistic regression may be a good method to compare the two groups retrospectively, however multiple other factors including sepsis, trauma, cardiac, renal respiratory disease, demographic profile all play a role in the choice of drug, the dosage administered, and subsequent hypotension. Standardization between two groups is best possible in prospective randomized control trials $(\mathrm{RCT})$ and planned emergency research should be the way forward in critically ill. Systematic review with more RCTs is advisable as a next step.

The secondary outcome noted was the first-pass intubation success during RSI while using etomidate vs ketamine as the induction agent. The result potentially reflects that both agents provide similar intubating conditions and allow timely placement of a definitive airway in acutely ill patients, in line with the goal of RSI.

The ability to intubate in the first attempt depends on multiple factors like underlying disease, difficult airway, airway aids, the expertise of personnel available, the setting of prehospital or inhospital, and also induction agent and muscle relaxant used. Safe and rapid securing of the airway is the primary goal of RSI.

Previous studies have focused on the effects of neuromuscular blockers as they are directly responsible for muscular relaxation for passage of the tracheal tube. ${ }^{11,12}$ However, the sedative used can affect this outcome by a variety of potential mechanisms. For instance, the response to the intubation stimulus, such as diaphragmatic movement and coughing, can be influenced by the sedative used. Also, the onset time of neuromuscular blockade can be modified by the sedative. This is particularly important in the context of our study because ketamine has a longer onset of effect compared to etomidate. ${ }^{11}$ Therefore, if intubation is attempted prior to maximal neuromuscular blockade, intubation success could be affected.

It is sensible to tailor the choice of induction agent to the need of the patient. An informed practitioner wisely employs the appropriate drug or a combination in the practice of good patient care. ${ }^{8}$ Clinicians should also prioritize preintubation resuscitation over selecting induction agents to optimize hemodynamics in all possible scenarios. It is also equally important that patients who are unstable or in shock despite attempts at optimization should receive lower induction doses. Appropriate drugs and advanced airway management techniques for the individual clinical scenario are the cornerstone for a successful RSI in an emergency setting. Familiarity with a range of induction agents is also important to avoid a single standardized induction approach for all patients.

\section{OrCID}

Bindu George (1) https://orcid.org/0000-0001-8801-0497

Nayanthara Joachim (1) https://orcid.org/0000-0002-9530-4423

\section{References}

1. Hagberg C. Hagberg and Benumof's airway management. 3th ed. Philadelphia: Elsevier; 2018. 273-275, 340-342.

2. Stept WJ, Safar P. Rapid induction-intubation for prevention of gastric contents aspiration. Anesth Analg 1970;49(4):633-636. PMID: 5534675.

3. Sinclair RCF, Luxton MC. Rapid sequence induction. Continuing Educ Anaesth Crit Care Pain 2005;5(2):45-48. DOI: 10.1093/bjaceaccp/ mki016.

4. Amornyotin S. Ketofol: a combination of ketamine and propofol. J Anaesthesia Crit Care 2014;1(5):00031. DOI: 10.15406/ jaccoa.2014.01.00031.

5. Shafer SL, Rathmell JP, Flood P. Stoelting's pharmacology and physiology in anesthetic practice. 5th ed. Philadelphia: Wolters Kluwer Health 2015;160-198.

6. Upchurch CP, Grijalva CG, Russ S, Collins SP, Semler MW, Rice TW, et al. Comparison of etomidate and ketamine for induction during 
rapid sequence intubation of adult trauma patients. Ann Emerg Med 2017;69(1):24-33. DOI: 10.1016/j.annemergmed.2016.08.009.

7. Morris C, Perris A, Klein J, Mahoney P. Anaesthesia in haemodynamically compromised emergency patients: does ketamine represent best agent? Anaesthesia 2009;64(5):532. DOI: 10.1111/j.1365-2044. 2008.05835.x.

8. Miller RD. Miller's anesthesia. 9th ed. Philadelphia: Churchill Livingstone/Elsevier 2019;1:748-786.

9. Jabre P, Combes X, Lapostolle F, Dhaouadi M, Ricard-Hibon A, Vivien $B$, et al. Etomidate versus ketamine for rapid sequence intubation in acutely ill patients: a multicentre randomised controlled trial. Lancet 2009;374(9686):293-300. DOI: 10.1016/S0140-6736(09)60949-1.
10. April MD, Arana A, Schauer SG, Davis WT, Oliver JJ, Fantegross $A$, et al. On behalf of the NEAR Investigators. Ketamine versus etomidate and peri-intubation hypotension: a national emergency airway registry study. Acad Emerg Med 2020;27(11):1106-1115. DOI: 10.1111/acem.14063.

11. Patanwala AE, McKinney CB, Erstad BL, Sakles JC. Retrospective analysis of etomidate versus ketamine for first-pass intubation success in an academic emergency department. Acad Emerg Med 2014;21(1):87-91. DOI: 10.1111/acem.12292.

12. Sharda SC, Bhatia MS. Etomidate compared to Ketamine for Induction during Rapid Sequence Intubation: Systematic Review and Metaanalysis. Indian J Crit Care Med 2021;26(1):108-113. 